\title{
On-chip quantum storage in a rare-earth-doped photonic nanocavity
}

\author{
Tian Zhong, Jonathan M. Kindem, Jake Rochman, Evan Miyazono, Andrei Faraon ${ }^{\mathrm{a}}$ and Alban \\ Ferrier, Philippe Goldner ${ }^{\mathrm{b}}$

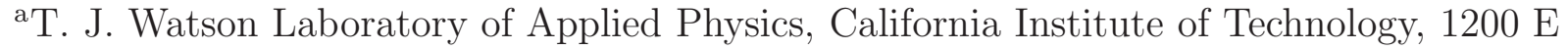 \\ California Blvd, Pasadena, CA, 91125, USA \\ ${ }^{\mathrm{b}}$ Institute de Chimie et des Matériaux Paris-Est, Univ. Pierre et Marie Curie, 11, rue Pierre et \\ Marie Curie, 75005 Paris, France
}

\begin{abstract}
Rare-earth-ion doped crystals are state-of-the-art materials for optical quantum memories and quantum transducers between optical and microwave photons. Here we describe our progress towards a nanophotonic quantum memory based on a rare-earth (Neodymium) doped yttrium orthosilicate (YSO) photonic crystal resonator. The Purcell-enhanced coupling of the $883 \mathrm{~nm}$ transitions of Neodymium $\left(\mathrm{Nd}^{3+}\right)$ ions to the nano-resonator results in increased optical depth, which could in principle facilitate highly efficient photon storage via cavity impedance matching. The atomic frequency comb (AFC) memory protocol can be implemented in the Nd:YSO nano-resonator by efficient optical pumping into the long-lived Zeeman state. Coherent optical signals can be stored and retrieved from the AFC memory. We currently measure a storage efficiency on par with a bulk crystal Nd:YSO memory that is millimeters long. Our results will enable multiplexed on-chip quantum storage and thus quantum repeater devices using rare-earth-ions.
\end{abstract}

Keywords: Quantum optics, Quantum information and processing, Quantum memories

\section{INTRODUCTION}

Quantum light-matter interfaces (QLMIs) are essential components in future quantum optical networks that reversibly map stationary qubits (such as in atoms, trapped ions or superconducting circuits) to photons. ${ }^{1}$ They will find applications for long-distance quantum communications, ${ }^{1}$ global distribution of clocks, ${ }^{2}$ and studies of fundamental physics. ${ }^{2}$ The QLMIs can be built from quantum light emitters with internal quantum states controllable by optical fields and coupled to photons. Scalable and robust QLMIs require emitters to have long spin coherence times and coherent optical transitions. Furthermore, in integrated optical quantum networks, these emitters need to be coupled to optical resonators that confine the photons in a single mode and further couple them into optical fibres. The solid-state emitters most investigated so far for on-chip QLMIs are semiconductor quantum dots $(\mathrm{QDs})^{8}$ and nitrogen vacancy centers in diamond $(\mathrm{NVs}){ }^{9}$ To date, complete quantum control of single QD and NV spins, spin-photon entanglement, and entanglement of distant NVs via photons have been realized. ${ }^{3,5,7}$ Both $\mathrm{QDs}^{4}$ and $\mathrm{NVs}^{6}$ have been coupled to optical nano-cavities. However, the challenge in growing optically identical QDs limits their usability for a scalable architecture. ${ }^{8}$ NVs embedded in nanostructures have long electronic spin coherence times, ${ }^{9}$ but suffer from optical spectral instabilities such as blinking and spectral diffusion. ${ }^{10}$ These spectral instabilities have made it difficult to coherently control NV centers via cavity field in nano-resonators.

REIs embedded in host crystals at cryogenic temperatures exhibit highly coherent quantum states in the $4 \mathrm{f}$ orbital. ${ }^{11}$ The hyperfine states of REIs can have spin coherence times as long as six hours, ${ }^{12}$ the longest ever demonstrated in a solid. These states are connected via optical transitions with the narrowest linewidth in the solid state (sub-kHz) and small inhomogeneous broadening (MHz to $\mathrm{GHz}) .{ }^{13}$ This outstanding optical and spin

Further author information: (Send correspondence to A. F. and T. Z. )

A. F. E-mail: faraon@caltech.edu

T. Z.: E-mail: tzhong@caltech.edu

Advances in Photonics of Quantum Computing, Memory, and Communication IX, edited by Zameer UI Hasan,

Philip R. Hemmer, Hwang Lee, Alan L. Migdall, Proc. of SPIE Vol. 9762, 97620J

(C) 2016 SPIE · CCC code: $0277-786$ X/16/\$18 · doi: 10.1117/12.2211999

Proc. of SPIE Vol. $976297620 \mathrm{~J}-1$ 
coherence makes REI-doped crystals the state-of-the-art material for macroscopic solid-state optical quantum memories. ${ }^{14-16}$ Integrated REI-doped waveguide quantum memories have also been developed. ${ }^{17}$ Detection and control of single REI spins has been recently demonstrated in bulk material, but not using the transitions employed in optical quantum memories. ${ }^{18,19}$

The integration of rare-earth ions in an on-chip quantum nanophotonic platform would enable multiplexed quantum repeaters and scalable quantum networks where REI ensembles act as quantum memories and single REIs act as qubits. ${ }^{20,26}$ Here we demonstrate optical storage in a Rare-earth-doped Yttrium orthosilicate $\mathrm{Y}_{2} \mathrm{SiO}_{5}$ (YSO) photonic crystal nano-beam resonator with small mode volume of 1.6 cubic wavelengths. The coupling of the $883 \mathrm{~nm}{ }^{4} \mathrm{I}_{9 / 2}{ }^{4} \mathrm{~F}_{3 / 2}$ transition of Neodymium $\left(\mathrm{Nd}^{3+}\right)$ ions to the nano-resonator resulted in an increased optical depth, which facilitates efficient photon storage via cavity impedance matching. ${ }^{21}$ Lifetime-limited optical coherence time $\mathrm{T}_{2}$ of $100 \mu \mathrm{s}$ at $3.6 \mathrm{~K}$ with low spectral diffusion was measured using photon echo technique. Our results point at a viable technology for on-chip quantum storage using rare-earth-ions.

a

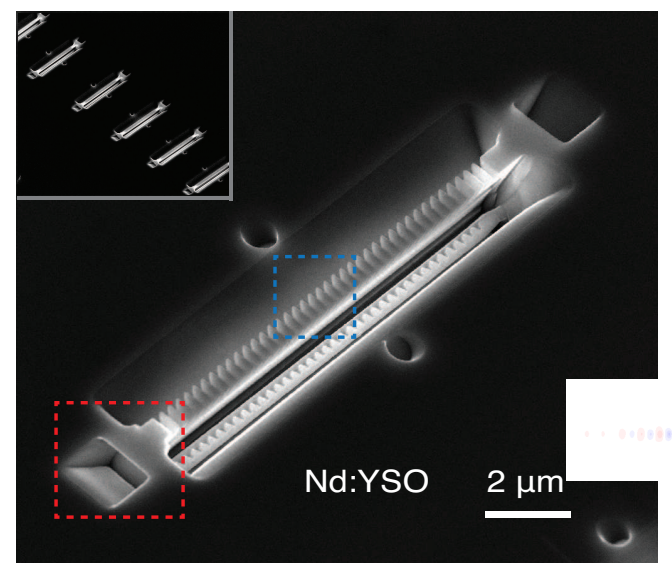

b

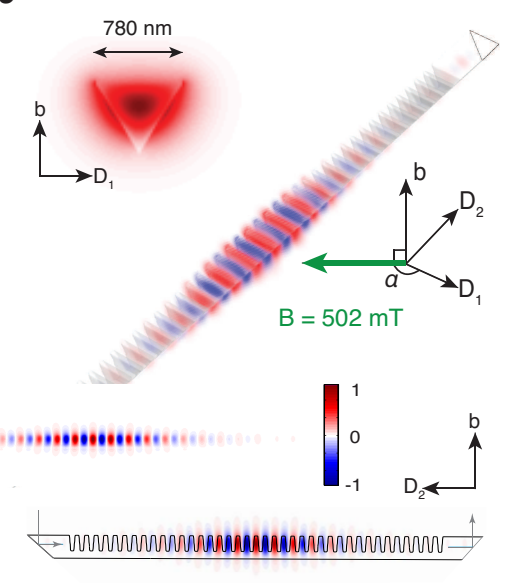

C

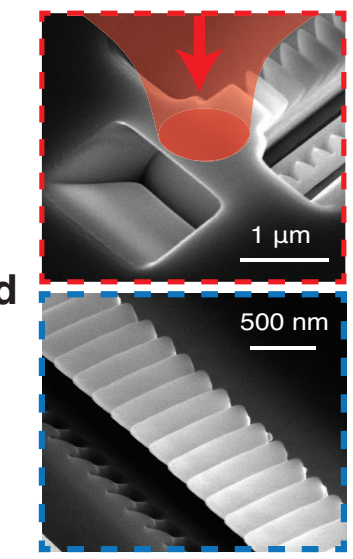

Figure 1. Scanning electron microscope images of the device. ${ }^{22}$ The red inset is a zoomed-in view of the $45^{\circ}$ angle-cut coupler that allows vertical coupling of light from a microscope objective. The blue inset shows the grooves forming the photonic crystal. Schematics of the resonator with simulated field profiles of the fundamental TE mode is also shown. The TE polarization aligns with the $\mathrm{D}_{1}$ axis of the YSO crystal. A magnetic field of $300 \mathrm{mT}$ is applied in the $\mathrm{D}_{1}-\mathrm{D}_{2}$ plane,typically at an angle of $\alpha=135^{\circ}$ with respect to $\mathrm{D}_{1}$ axis.

\section{DESIGN AND FABRICATION OF YSO NANOPHOTONIC RESONATORS}

Many photonic crystal cavity designs use circular perforations in the center of a 1-D nanobeam to create a photonic bandgap and use modulations of the perforations geometry to obtain a local cavity mode within the structure. When using focused ion beam milling, this method presents difficulty for making high-Q cavities largely due to misalignment of the perforations with respect to the nanobeam axis. To minimize this issue, a photonic crystal cavity design consisting of a triangular nanobeam with a lattice of sub-wavelength grooves is proposed. The pattern of grooves on the triangular beam is milled across the entire beam width, which eases the alignment of the grooves to the nanobeam. Another benefit of the triangular nanobeam design is that all its dimensions can be globally scaled to attain resonances in a wide spectral range on the same chip. This flexibility is generally less available for photonic devices fabricated on thin films, for which a different thin film thickness is required to match specific resonance wavelengths.

We fabricated triangular nano-beam resonator in YSO using focused ion beam milling. ${ }^{22-25,28}$ The SEM images of the Nd:YSO devices are shown in Fig. 1. Two coupling ports were milled at both ends of the beam at $45^{\circ}$ with respect to the sample surface to permit broadband excitation and collection through the cavity mirrors. 
The coupling efficiency of each coupler was $\sim 20 \%$ on average. The cavity transmission signal was collected vertically using a confocal microscope setup. We also fabricated resonators of varying dimensions to operate at visible, near infrared and telecom wavelengths. ${ }^{23}$ They were designed to couple to different rare-earth ions including Europium, Praseodymium, and Erbium. Figure 2 (a-c) shows the simulated field profile of transverse electric (TE) polarization resonance mode, which are identical for all the devices at different wavelengths. Figure 2(d) shows typical TE broadband transmission spectrum of a YSO nanobeam resonators showing a resonance in the photonic bandgap. Figure 2(e-g) plot the resonances close to the target atomic transition of Pr, Nd, andf Er ions. The measured resonance quality factors range from 3,000 to 27,000, which are highest reported to date for devices fabricated using focused ion beam. ${ }^{23}$

\section{COUPLING OF RARE-EARTH DOPANTS TO A YSO PHOTONIC CRYSTAL CAVITY}

The cavity resonance initially at $880 \mathrm{~nm}$ was tuned to $883 \mathrm{~nm} \mathrm{Nd}$ transition by gas injection into the cryostation while the sample was cooled to $3.6 \mathrm{~K}$. When coupled, enhanced photoluminescence and reduced lifetime from $290 \mu \mathrm{s}$ (cavity off resonance) to $70 \mu \mathrm{s}$ were observed. The lifetime in bulk samples was $250 \mu \mathrm{s}$. Factoring in the branching ratio of the transition of $5 \%$, this corresponds to an ensemble averaged spontaneous emission rate enhancement (Purcell Factor) F 70. The maximum enhancement for ions positioned in the maximum field in the cavity is then estimated to be $\mathrm{F}=200$, based on the fundamental mode field profile given in Fig. 2(a-c).

\section{OPTICAL STORAGE BASED ON STIMULATED PHOTON ECHOES}

Two-pulse photon echo experiments were performed to extract optical coherence time $\mathrm{T}_{2}$ of the Nd ions in the cavity. We measured $\mathrm{T}_{2} \sim 100 \mu \mathrm{s}$ for the coupled ions in the nano-resonator at $3.6 \mathrm{~K}$ temperature with 300 $\mathrm{mT}$ applied magnetic field, which agrees well with the $\mathrm{T}_{2}$ measured in the bulk sample. We then demonstrated multi-mode optical storage in our nanophotonic cavity using stimulated photon echoes. ${ }^{25,27}$ An arbitrary 3-bit pulse sequence (i.e. 101 or 110) followed by write and read pulses was sent into the cavity. As shown in Fig. 3, multiple photon echoes resembling the time-reversed input pulse sequence were emitted after the read pulse. An additional echo at $7 \mu \mathrm{s}$ was produced by the write and read pulses, which did not contribute to the data storage. Current data pulse widths were limited to 200 ns by the rise time of our acousto-optic modulators (AOM). In principle, by using much narrower pulses ( $\sim 10 \mathrm{~ns})$, storage of longer bit length ( $\sim 6 \mathrm{bit})$ can be implemented using our device.

\section{QUANTUM STORAGE BASED ON ATOMIC FREQUENCY COMBS}

Furthermore, we are implementing atomic frequency combs (AFC) storage in the nano-resonator. First, the ground state was Zeeman split by $20 \mathrm{GHz}$ using $300 \mathrm{mT}$ applied magnetic field. An AFC was prepared by exciting the cavity with a train of $0.7-\mu$ s intense pulses for $1 \mathrm{~ms}$, which frequency-selectively removed ions from the ground state. The AFC was prepared by optically pumping the ions into the long-lived ( $\sim 60 \mathrm{~ms}$ lifetime at $3.5 \mathrm{~K}$ ) Zeeman state. Note that the optical pumping efficiency, measured by the percentage of ions that can be removed from the ground state to the Zeeman storage state, is strongly determined by the ratio of the Zeeman lifetime to the excited state lifetime. A higher ratio increases the optical pumping efficiency. The Purcell enhancement in the Nd:YSO device resulted in a significantly shortened excited state lifetime, which helped to achieve an efficient optical pumping and higher contrast of the frequency comb. The frequency spacing of the comb was $1-4 \mathrm{MHz}$, resulting in a storage time of $250 \mathrm{~ns}$ to $1 \mu$ s with low noise. Coherent pulses attenuated to the single photon level were used to characterize the quantum memory. We currently measure a storage efficiency on par with a bulk crystal Nd:YSO memories that is millimeters long. A memory-compatible entangled photon source based on a Lithium Niobate thin sheet cavity could in principle interface well with this Nd:YSO device. High-fidelity quantum storage of the entangled state has been demonstrated in bulk AFC memories ${ }^{29}$ which is expected to work with our nanocavity device. 

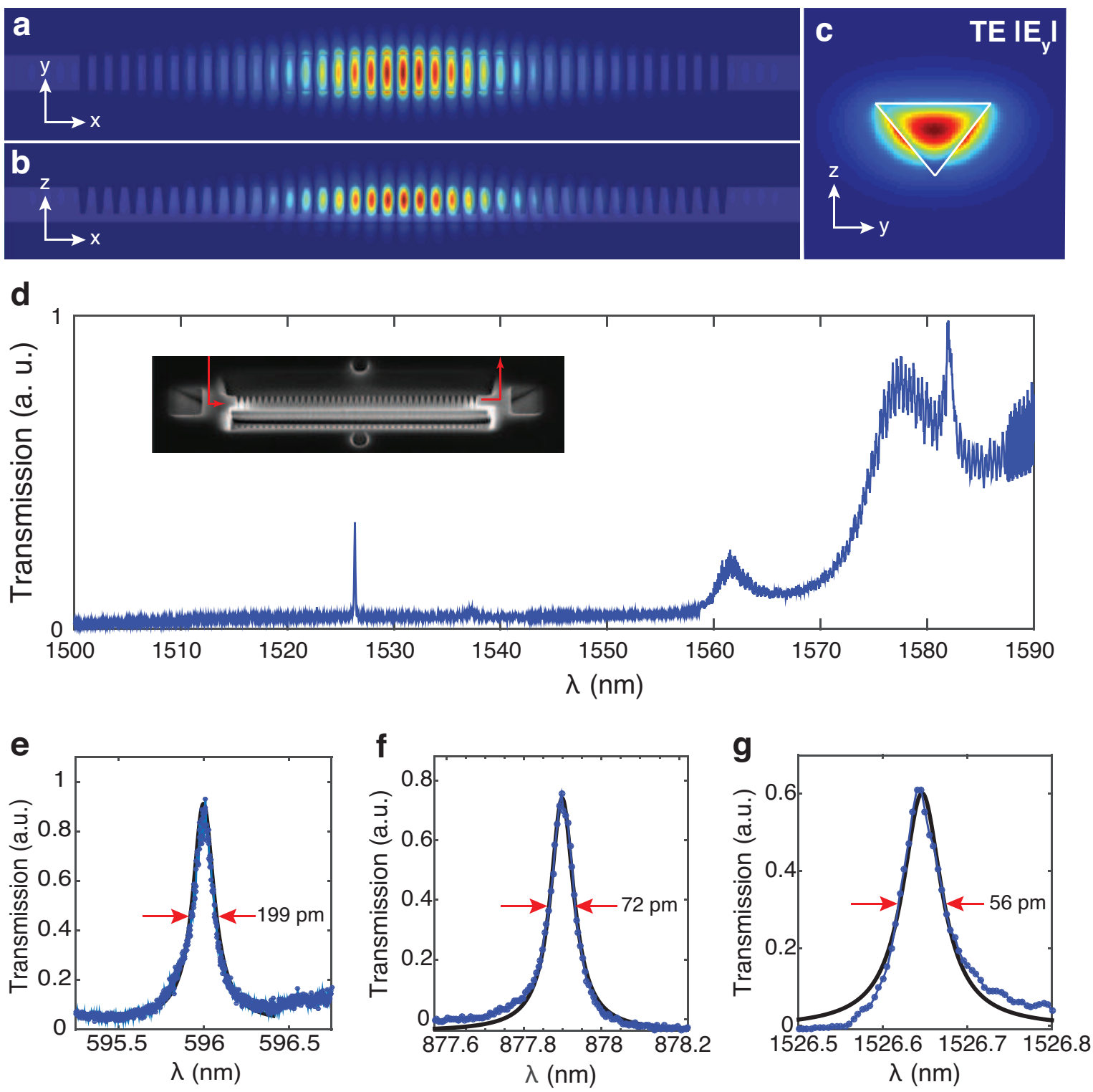

Figure 2. Nanobeam resonators in the YSO crystal. ${ }^{23}$ (a-c) Top, side and cross-section views of the simulated mode profiles of the TE-mode resonance. (d) Typical TE broadband transmission spectrum of a YSO nanobeam resonators showing a resonance in the photonic bandgap. Inset shows the transmission measurement scheme in which broadband super-continuum light vertically couples into the nanobeam from one end and is collected from the other end. (e) Resonance close to the target $605 \mathrm{~nm}$ atomic transition of Pr ions. (f) Resonance close to the $883 \mathrm{~nm}$ transition of Nd ions. (g) Resonance close to the $1536 \mathrm{~nm}$ transition of Er ions.

\section{ACKNOWLEDGMENTS}

This work was funded by California Institute of Technology (Caltech) and National Science Foundation (NSF) CAREER award number 1454607. Equipment funding was also provided by the Institute of Quantum Information and Matter (IQIM), an NSF Physics Frontiers Center with support of the Moore Foundation. The device nanofabrication was performed in the Kavli Nanoscience Institute at Caltech. 

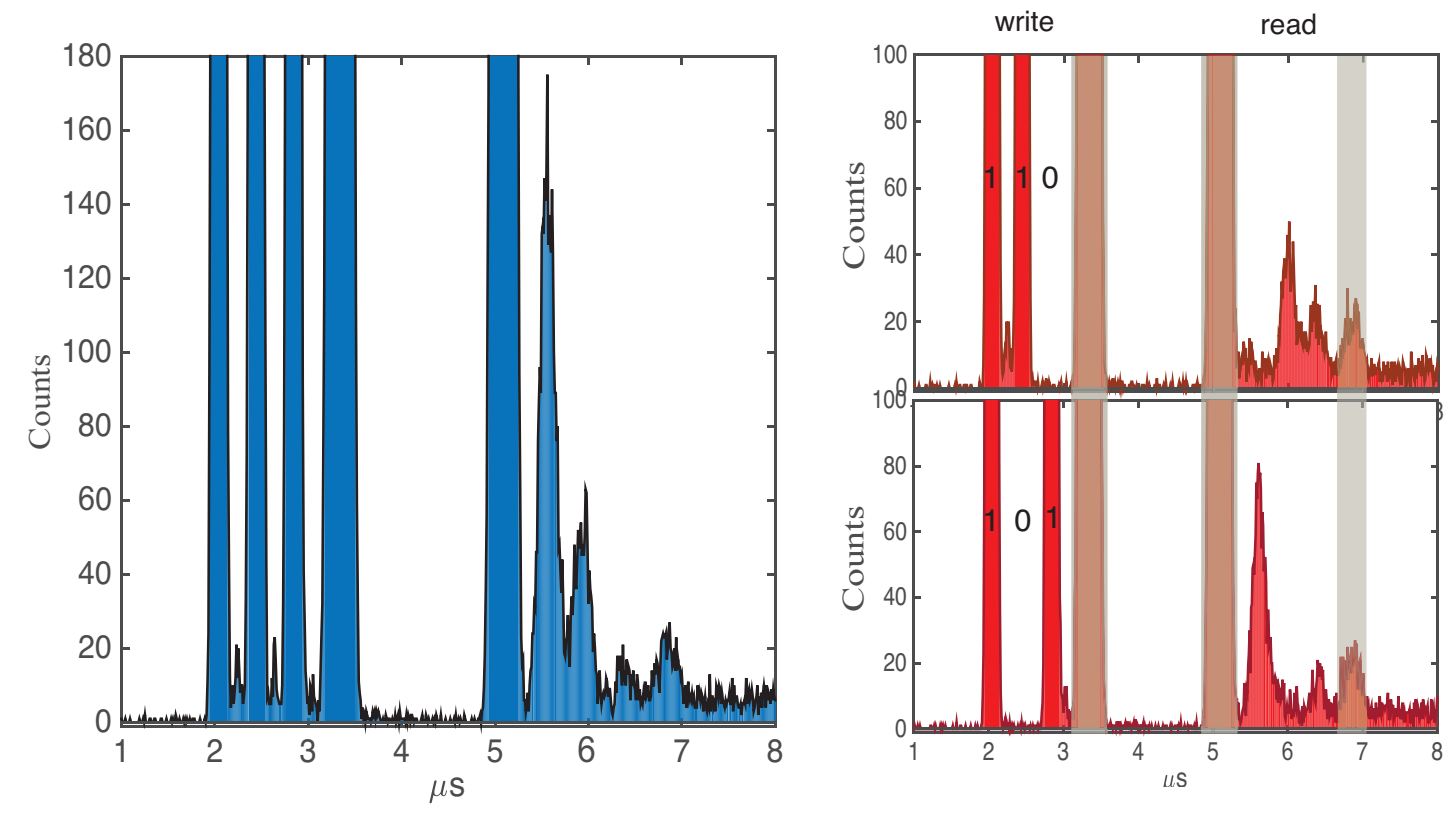

Figure 3. Optical data storage in the Nd:YSO nano-resonator based on stimulated photon echoes. Ignoring the last echo at $7 \mu \mathrm{s}$, the echo amplitudes represent a time-reserved sequence of the input signals.

\section{REFERENCES}

[1] Kimble, H. J. "The quantum internet." Nature 453, 1023-1030 (2008).

[2] Kómár, P., Kessler, E. M., Bishof, M., Jiang, L., Sørensen, A. S., Ye, J., and Lukin, M. D. "A quantum network of clocks." Nature Phys. 10, 582-587 (2014).

[3] Press, D., Ladd, T. D., Zhang, B., and Yamamoto Y. "Complete quantum control of a single quantum dot spin using ultrafast optical pulses." Nature 456, 218-221 (2008).

[4] Englund, D., Faraon, A., Fushman, I., Stoltz, N., Petroff, P., and Vucković,, J. "Controlling cavity reflectivity with a single quantum dot." Nature 450, 857-861 (2007).

[5] De Greve, K., et al. "Quantum-dot spin-photon entanglement via frequency downconversion to telecom wavelength." Nature 491, 421-425 (2012).

[6] Faraon, A., Barclay, P. E., Santori, C., Fu, K. C., and Beausoleil, R. G. "Resonant enhancement of the zero-phonon emission from a colour centre in a diamond cavity." Nature Photon. 5, 301-305 (2011).

[7] Bernien H., et al. "Heralded entanglement between solid-state qubits separated by three metres." Nature 497, 7447 (2013).

[8] Michler, P. (Ed.) Single Quantum Dots (Springer Berlin Heidelberg, 2009).

[9] Aharonovich, I., and Neu, E. "Diamond Nanophotonics." Adv. Optical Mater. 2, 911-928 (2014).

[10] Faraon., A., Santori, C., Huang, Z., Acosta, V. M., and Beausoleil, R. G. "Coupling of Nitrogen-Vacancy Centers to Photonic Crystal Cavities in Monocrystalline Diamond." Phys. Rev. Lett. 109, 033604 (2012).

[11] Thiel, C., Böttger, T., and Cone, R. "Rare-earth-doped materials for applications in quantum information storage and signal processing." J. Luminesc. 131, 353-361(2001).

[12] Zhong, M., Hedges, M. P., Ahlefeldt, R. L., Bartholomew, J. G., Beavan, S. E., Wittig, S. M., Longdell, J. J., and Sellars, M. J. "Optically addressable nuclear spins in a solid with a six-hour coherence time." Nature 517, 177-180 (2015).

[13] Sun, Y., Thiel, C. W., Cone, R. L., Equall, R. W. and Hutcheson, R. L. "Recent progress in developing new rare earth materials for hole burning and coherent transient applications." J. Lumin. 98, 281-287 (2002). 
[14] Lvovsky, A. I., Sanders, B. C., and Tittel, W. "Optical quantum memory" Nature Photon. 3, 706-714 (2009).

[15] Tittel, W., Afzelius, M., Chaneliére, T., Cone, R. L., Kröll, S., Moiseev, S. A., and Sellars, M. "Photon-echo quantum memory in solid state systems." Laser 83 Photon. Rev. 4, 244-267 (2010).

[16] de Riedmatten, H., Afzelius, M., Staudt, M. U., Simon, C., and Gisin, N. "A solid-state light-matter interface at the single-photon level." Nature 456, 773-777 (2008).

[17] Saglamyurek, E., et al. "Broadband waveguide quantum memory for entangled photons." Nature 469, $512-515$ (2011).

[18] Kolesov R. et al. "Optical detection of a single rare-earth ion in a crystal." Nature Commun. 3, 1029 (2012).

[19] Utikal, T. et al. "Spectroscopic detection and state preparation of a single praseodymium ion in a crystal." Nature Commun. 5, 3627 (2014).

[20] McAuslan, D. L., and Longdell, J. J. "Cavity QED using rare-earth-metal-ion dopants in monolithic resonators: What you can do with a weak oscillator." Phys. Rev. A 80, 062307 (2009).

[21] Afzelius, M., and Simon, C. "Impedance-matched cavity quantum memory." Phys. Rev. A 82, 022310 (2010).

[22] Zhong, T., Kindem, J., Miyazono, E., and Faraon, A. "Nanophotonic coherent light-matter interfaces based on rare-earth doped crystals." Nat. Commun. 6, 8206 (2015).

[23] Zhong, T., Rochman, J., Kindem, J., Miyazono, E., and Faraon, A. "High quality factor nanophotonic resonators in bulk rare-earth doped crystals." Opt. Express 24, 536-544 (2016).

[24] Zhong, T., Hartz, A., Miyazono, E., and Faraon, A. "Towards Coupling Rare Earth Ions to a $\mathrm{Y}_{2} \mathrm{SiO}_{5}$ Nanophotonic Resonator." CLEO: 2014 OSA Technical Digest (online), paper FTh1B.5 (Optical Society of America, 2014).

[25] Zhong, T., Kindem, J., Miyazono, E., and Faraon, A. "Nanophotonic Quantum Memory Based on RareEarth-Ions Coupled to an Optical Resonator." CLEO: 2015 OSA Technical Digest (online), paper FW3A.3 (Optical Society of America, 2015).

[26] Zhong, T., Kindem, J., Miyazono, E., and Faraon, A., "Towards Detection of Single Rare-Earth-Ions in a Nanophotonic Resonator." CLEO: 2015 OSA Technical Digest (online), paper FTh4B.7 (Optical Society of America, 2015).

[27] Zhong, T., Kindem, J., Miyazono, E., and Faraon, A., "Nanophotonic photon echo memory based on rare-earth-doped crystals" APS Meeting Abstracts 1, 37003( 2015).

[28] Miyazono, E., Zhong, T., Craiciu, I., Kindem, J., and Faraon, A. "Coupling of erbium dopants to yttrium orthosilicate photonic crystal cavities for on-chip optical quantum memories." Appl. Phys. Lett. 1, 01111 (2016).

[29] Clausen, C. et al. "Quantum storage of photonic entanglement in a crystal." Nature 469, 508-511 (2011). 